\title{
Implementación y pruebas de sistema de inyección de agua en un vehículo dedicado a gas natural como estrategia para reducir problemas asociados a la alta temperatura en la cámara de combustión
}

\author{
Implementation and testing of water injection system in a vehicle \\ dedicated to natural gas as a strategy to reduce problems associated with \\ high temperature in the combustion chambers
}

\author{
Luis Carlos Rios Quiroga, Luis Guillermo Gaviria Arboleda, Cesar Augusto Borrero Gómez \\ Facultad de Ingeniería Mecánica, Universidad Tecnológica de Pereira, Risaralda, Colombia. \\ Correo-e: lcriosq@gmail.com
}

\begin{abstract}
Resumen- Un reconocido concesionario tiene varios vehículos utilitarios, de la marca que representa, que usa en su red de distribución de piezas de repuesto y servicios de mecánica vehicular. Estos vehículos funcionan con gas natural y venían presentando una falla cíclica en el empaque de culata del pistón numero cuatro debido a la alta temperatura que se genera en la cámara de combustión. Esta falla conlleva no sólo problemas mecánicos, sino también en la logística de distribución.

Se propuso emplear un dispositivo de inyección de agua, agua etanol y agua - metanol para reducir la temperatura en la cámara de combustión y verificar que no se vea afectado el desempeño del motor, a través de pruebas de desempeño en un dinamómetro de cubos. Los resultados muestran que se logra disminuir la temperatura sin sacrificar desempeño en el motor.
\end{abstract}

Palabras clave- Inyección de agua, metanol, etanol, dinamómetro.

Abstract - A recognized dealership has some utilitarian vehicles, of brand that he represents, which uses in its distribution network of spare parts and mechanical vehicular services. These vehicles run with natural gas and have been presenting cyclical fails in packaging of number four piston head due to high temperature that is generated in the combustion chamber. This fault involves not only mechanical problems, but also in distribution logistics. Was proposed employ a device water injection, water - ethanol and water - methanol to reduce the temperature in the combustion chamber and verifying that not affected engine performance, through performance tests on a cubes dynamometer. The results show that the temperature decrease is achieved without sacrificing performance in the engine
Key Word - Injecting water, methanol, ethanol, dynamometer.

\section{INTRODUCCIÓN}

Actualmente la empresa Chevrolet Caminos, concesionario de la marca para Risaralda, Quindío y Norte del Valle, cuenta con una flota de cinco vehículos de carga, de los cuales tres son Chevrolet Super Carry convertidos para trabajar exclusivamente con Gas Natural Vehicular, con el fin de obtener una economía del $50 \%$ en los costos del combustible y para evitar los mantenimientos frecuentes en la bomba de combustible y carburador que estos vehículos demandaban con la operación a gasolina.

En el funcionamiento de la flota y a través de las operaciones correctivas de mantenimiento es detectada una falla repetitiva. Este inconveniente, asociado a un recalentamiento en el motor, debido a la alta temperatura alcanzada en la cámara de combustión, genera la ruptura del empaque de culata del motor en su cilindro número cuatro, que genera el desmonte de la culata, el cambio del empaque y el cambio de los gorros de válvula, incide vitalmente en la operación de la compañía, generando la restructuración logística en el momento que sucede, replanteando sus rutas de entrega, horas adicionales de reparación, solicitud de apoyo de otras áreas, entre otros. Por lo tanto esta falla es identificada, con un seguimiento durante un año, analizando su frecuencia por parte del Coordinador Logístico de Repuestos de la Compañía quien decide buscar alternativas que contribuyan a la eliminación de esta falla, planteando una investigación en conjunto con el 
Laboratorio de Pruebas Dinámicas Automotrices (LPDA) de la Universidad tecnológica de Pereira.

El problema de recalentamiento con vehículos que utilizan Gas Natural es generalizado, pues existen evidencias de daños similares en otras marcas y líneas de vehículos dedicados a trabajar con gas natural, con daños en culata, empaque de culata, gorros de válvulas y otros componentes, debido a la alta temperatura que se genera en la cámara de combustión al utilizar este tipo de combustible.

La propuesta de investigación consta de la instalación de un dispositivo de inyección de agua en uno de los vehículos, en el que se estudiaran diferentes líquidos refrigerantes como son: agua, agua - etanol y agua - metanol. Sin olvidar que esto puede traer consecuencias perjudiciales al desempeño del motor en su funcionamiento.

La metodología utilizada está basada en la aplicación de tecnología de inyección de agua disponible en el mercado, haciendo uso de la experiencia y el conocimiento de la falla mencionada, que a través del manejo de principios mecánicos y el apoyo del Laboratorio de Pruebas Dinámicas Automotrices de la Universidad Tecnológica de Pereira.

Para probar la influencia del sistema de inyección de agua en el vehículo de prueba, se harán mediciones de la temperatura de los gases de escape, como medida indirecta de la temperatura de la cámara de combustión, tanto del vehículo funcionando únicamente con Gas Natural, como con la adición de agua y agua-etanol en la admisión del motor a través del sistema de inyección que se implementará. Igualmente se harán mediciones de potencia para observar la incidencia de la inyección del agua y agua-etanol en esta variable de desempeño.

\section{CONTENIDO}

\section{Marco teórico.}

En 1920 durante el desarrollo de la aviación con fines militares en los Estados Unidos, se diseñaron y utilizaron dispositivos de inyección de agua en los motores de los aviones en busca de reducir y controlar la temperatura, debido a que se presentaban tendencias al sobrecalentamiento, producto de las largas horas de funcionamiento y las exigentes maniobras de estas aeronaves.

Durante el funcionamiento de estos dispositivos con fines netamente de control de temperatura en este tipo de motores, se detectaron las mejoras en potencia de los mismos, llegando a subir niveles de eficiencia térmica de estos motores de combustión interna; pero quedando rezagados durante varios años debido al cambio de tecnología de motores de pistón por turbinas que fueron desarrolladas al límite de rendimiento que exigía el desarrollo militar de los norteamericanos.

Solo hasta los años 80, en los Estados Unidos personas interesadas en las competencias automovilísticas y buscando hacer más eficientes los motores disponibles del mercado, sin depender sólo de los motores de alto cilindraje y de gran caballaje, desarrollados desde los años 50, empezaron a retomar e implementar dispositivos como turbocargadores de la mano con sistemas de inyección de agua, llegando a obtener valores de un 50\% más caballos de fuerza, al realizar pequeñas modificaciones en los vehículos de línea que se conseguían en el mercado durante la época.

De esta misma manera el mercado interesado en competencias de automóviles, empezó a probar mezclas de combustibles alternos junto con la gasolina en busca de aún mejores rendimientos, encontrando que la inyección de agua-metanol o solo metanol, no solo presentaba mejoras importantes en la temperatura de los motores, sino que también mejoraba representativamente los caballos de fuerza entregados por el mismo, debido a las características de este combustible, reduciendo representativamente el desgaste interno de las piezas; es decir alargando la vida útil de los motores.

\section{a- Motor de combustión interna}

Un motor de combustión interna, o motor a explosión o motor a pistón, es un tipo de máquina que obtiene energía mecánica directamente de la energía química de un combustible que arde dentro de una cámara de combustión. Su nombre se debe, a que dicha combustión se produce dentro de la máquina en si misma.

\section{b- Cámara de combustión}

La cámara de combustión es un cilindro, por lo general fijo, cerrado en un extremo y dentro del cual se desliza un pistón muy ajustado al cilindro. La posición hacia dentro y hacia fuera del pistón modifica el volumen que existe entre la cara interior del pistón y las paredes de la cámara. A través de una biela conectada al cigüeñal, convierte en movimiento rotatorio el movimiento lineal del pistón.

\section{c- Refrigeración del motor}

La refrigeración en motores de combustión interna debe hacerse para eliminar el exceso de calor generado durante el funcionamiento de éstos [1].

La refrigeración implica transferir la energía del cuerpo, aprovechando sus propiedades termodinámicas. $\mathrm{La}$ temperatura es el reflejo de la cantidad o nivel de energía que posee el cuerpo, ya que el frío propiamente no existe, los cuerpos sólo tienen más o menos energía térmica. De esta manera enfriar corresponde a retirar Energía.

Durante la combustión, parte de la energía generada no es convertida en energía mecánica y se pierde en forma de calor. Según la velocidad de giro del motor y el diseño de éste, sólo alrededor del $30 \%$ de la energía almacenada en el combustible se aprovecha para realizar trabajo mecánico, y el resto es necesario eliminarlo para evitar que la temperatura alcance valores críticos que puedan comprometer la integridad física del motor. 


\section{d- Sistema inyección de agua}

Los sistemas de inyección de agua han sido utilizados a lo largo de la historia en diferentes áreas de la industria, como lo son en las turbinas de aviación, en equipos para la generación de energía como calderas y en aplicaciones puntuales del área automotriz. El dispositivo utilizado para este proyecto es de un funcionamiento sencillo, basado en mecánica y electrónica básica, el cual fue adquirido en el mercado e instalado por la compañía Chevrolet Caminos, bajo la supervisión y el direccionamiento del Laboratorio de Pruebas Dinámicas Automotrices de la Universidad Tecnológica de Pereira.

El sistema de inyección cuenta con los siguientes elementos:

- Bomba de eléctrica de 12 V y 100 psi.

- Depósito para mezcla refrigerante de 1 galón.

- $\quad$ Tobera de inyección de 0,9 galones por hora.

- Válvula de regulación de presión con manómetro.

Todo viene montado en una pequeña caja de la marca SQUIRTLAB.

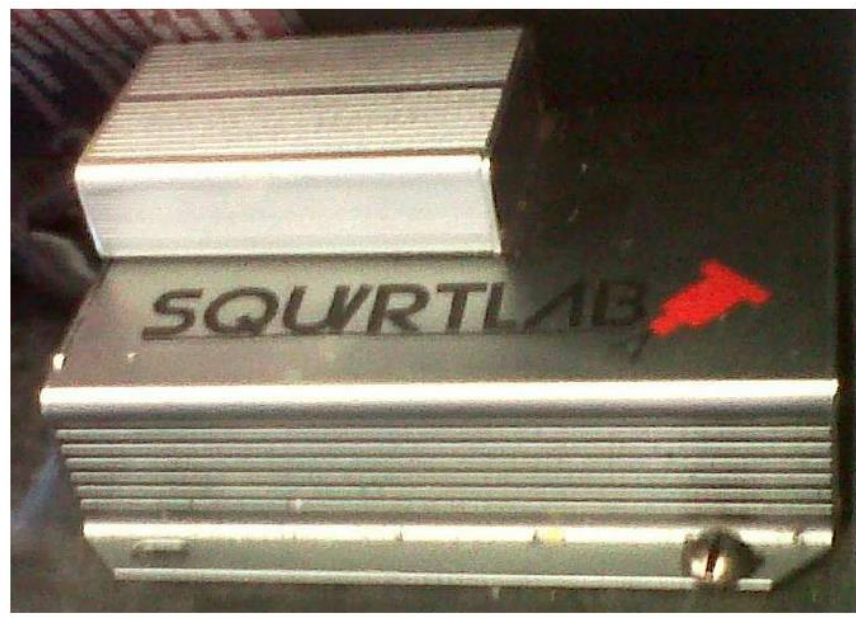

Figura 1. Caja con bomba eléctrica y sistema electrónico.

e- Descripción técnica del vehículo.

En la tabla 1 se muestras las características técnicas del vehículo.

\begin{tabular}{|c|c|}
\hline \multicolumn{2}{|c|}{ FICHA TECNICA } \\
\hline Marca & CHEVROLET \\
\hline Modelo & SUPER CARRY \\
\hline Placa & ZRM045 \\
\hline Año & 2000 \\
\hline Motor & 4 cilindros en línea OHC \\
\hline Cilindraje & $970 \mathrm{cc}$ \\
\hline Número Válvulas & 8 \\
\hline Potencia & $45 \mathrm{HP} @ 5300 \mathrm{rpm}$ \\
\hline Torque & $75 \mathrm{Nm} @ 3200 \mathrm{rpm}$ \\
\hline Admision & Carburador \\
\hline Combustible & Discos \\
\hline Frenos Delanteros & Tambor \\
\hline Frenos Traseros & $1410 \mathrm{~kg}$ \\
\hline Peso Bruto & $785 \mathrm{~kg}$ \\
\hline Capacidad Carga &
\end{tabular}

Tabla 1. Ficha técnica Chevrolet super carry.

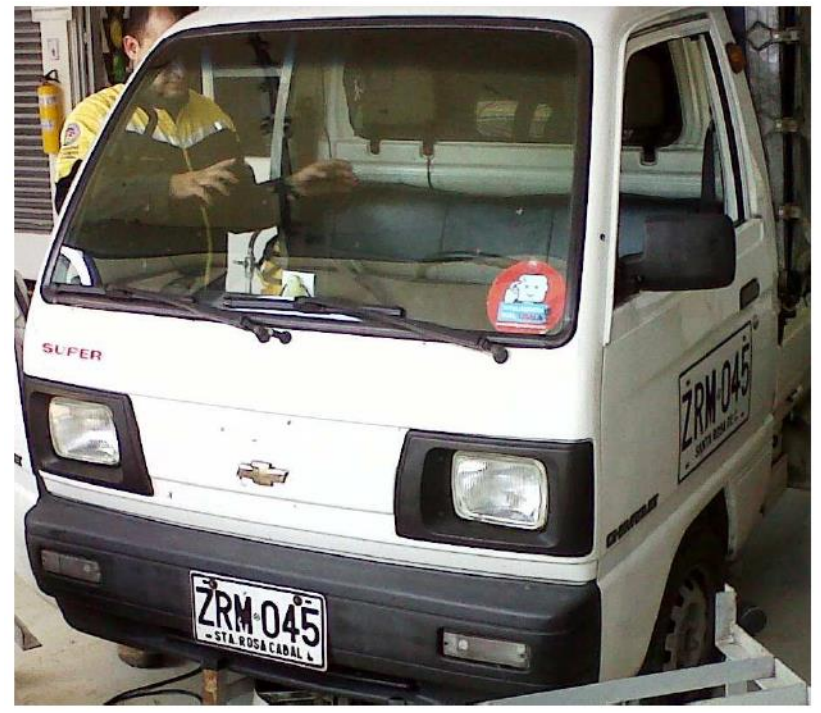

Figura 2. Vehículo usado en las pruebas

f- Dinamómetro de cubos

Para determinar la potencia del motor se hizo uso del dinamómetro de cubos, el cual permite determinar el comportamiento de esta variable en función del rango de revoluciones del motor. De igual manera el dinamómetro permite determinar el comportamiento del par en el rango de revoluciones del motor.

El dinamómetro toma directamente la potencia del acople de las ruedas de tracción del vehículo, con lo que la potencia medida es la que el vehículo tiene en las ruedas y en las condiciones ambientales locales, es decir la potencia efectiva. Esta potencia es menor que la que viene especificada de catalogo ya que ésta última corresponde a la potencia que desarrolla directamente el motor en condiciones ideales y no tiene en cuenta las perdidas en los elementos de trasmisión y por las condiciones ambientales. 


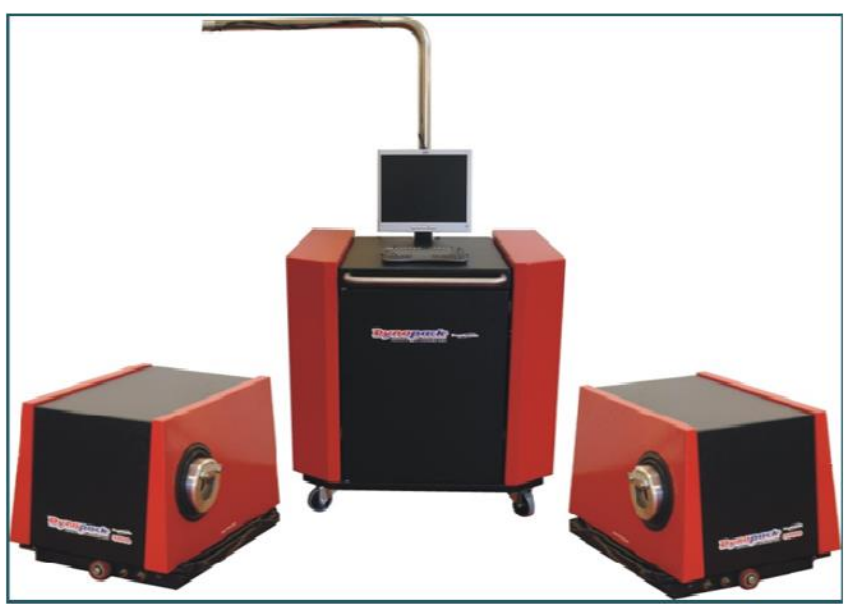

Figura 3. Dinamómetro de cubos.

2. Montaje, pruebas y análisis de resultados

Las pruebas a realizadas al vehículo se llevaron a cabo en dos fases, en la primera se toman las características y comportamiento del vehículo antes de instalar el dispositivo y la segunda etapa de pruebas con el dispositivo funcionando, haciendo uso de los tres refrigerantes que son agua, mezcla de agua-etanol y mezcla de agua-metanol.

Se prepararon mezclas al $50 \%$ es decir, mitad de agua y mitad de etanol o metanol.

\section{a- Instalación}

La instalación del dispositivo fue realizada por parte del proveedor Squirtlab en las instalaciones de Chevrolet Caminos, actividad desarrollada en tres sesiones de cuatro horas cada una, en donde se aprovecharon los espacios disponibles en el vehículo para instalar la bomba, el tanque de almacenamiento, el manómetro, la tubería de alimentación, el módulo electrónico, el interruptor y el circuito eléctrico.

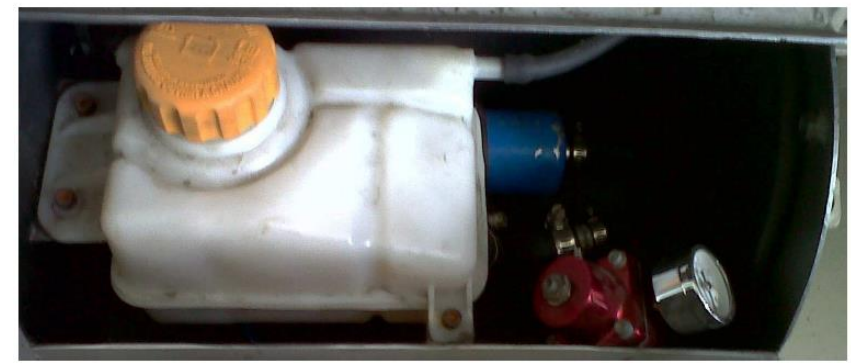

Figura 4. Sistema de inyección.

La boquilla de pulverización del sistema de inyección se instaló en el ducto de admisión de aire, entre el filtro y el punto de inyección de gas.

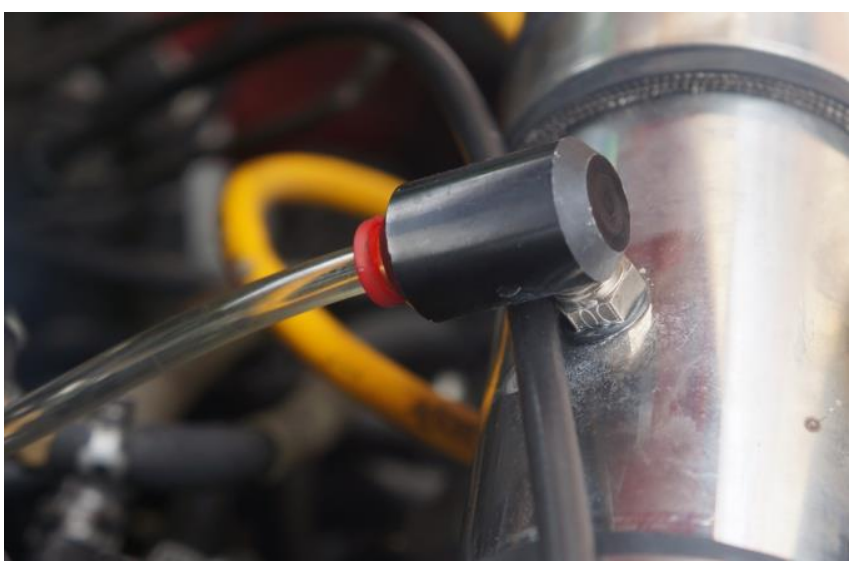

Figura 5. Boquilla de aspersión del sistema de inyección.

Para la medición de la temperatura en los gases de escape se usó un sensor instalado en el tubo de unión del múltiple de escape del vehículo. Se realizó en la parte más cercana a la cámara de combustión para garantizar la calidad de la medición, disminuyendo de esta manera las pérdidas y desviaciones a que diera lugar.

\section{b- Puesta a punto}

Después de tener el sistema completamente instalado se debe poner a punto, por lo tanto se ajustó la inyección de agua para que suministre dosis ideal y el avance de encendido requerido en toda la gama de operación del vehículo, este procedimiento se conoce como programación. También se requiere configurar la función de rangos de operación, entre las 1500 rpm y $4500 \mathrm{rpm}$, para que la bomba pueda operar en unos rangos seguros que no afecten la combustión del motor. En bajas revoluciones la inyección de agua provocaría un mal funcionamiento en la combustión del motor y en los rangos de bajas revoluciones no existe el problema de alta temperatura. Igualmente es necesario probar todos los elementos y si se presentan inconvenientes buscar las soluciones adecuadas. Todo este procedimiento fue llevado a cabo por parte del proveedor Squirtlab.

Es de anotar que se debe regular la presión del sistema para alcanzar una pulverización adecuada del líquido refrigerante.

\section{c- Prueba sin sistema de inyección}

Se realizaron dos pruebas, para cada fluido refrigerante, con seis repeticiones cada una; donde se registró el régimen de revoluciones contra potencia, la relación aire combustible, la presión de admisión y la temperatura a la salida de los gases de escape.

Para hacer la prueba se deja el vehículo a un régimen constante de 3000 revoluciones por minuto y se acelera a plena carga, se espera a que se estabilice la temperatura de trabajo del motor y se toman los datos sin disminuir la carga.

Para hacer esta prueba es necesario que el vehículo esté montado en el dinamómetro, que es el que se encarga de 
mantener fijo el régimen de giro del motor a pesar de tener el acelerador a fondo.

Debido al poco espacio sólo se mostrarán los resultados de una de las dos pruebas y al final se mostrarán los promedios de las dos pruebas y sus repeticiones.

\begin{tabular}{|c|c|c|c|c|c|c|}
\hline \multicolumn{6}{|c|}{ Resultados sin corrección prueba 2 } & \multicolumn{3}{|c|}{ Gas } \\
\hline & $\begin{array}{c}\text { Potencia } \\
\text { maxima (hp) }\end{array}$ & $\begin{array}{c}\text { Régimen } \\
\text { máxima } \\
\text { potencia (rpm) }\end{array}$ & $\begin{array}{c}\text { Régimen } \\
\text { máxima } \\
\text { potencia }(\mathrm{rpm} \\
\mathrm{x} 100)\end{array}$ & $\begin{array}{c}\text { Par Torsor } \\
\text { máximo (Nm) }\end{array}$ & $\begin{array}{c}\text { Régimen } \\
\text { máximo par } \\
(\mathrm{rpm})\end{array}$ & $\begin{array}{c}\text { Régimen } \\
\text { máximo par } \\
(\mathrm{rpm} \times 100)\end{array}$ \\
\hline corrida 1 & 14,1 & 3613 & 36,13 & 155,5 & 2381 & 23,81 \\
\hline corrida 2 & 14.1 & 3840 & 38,4 & 163,9 & 1502 & 15,02 \\
\hline corrida 3 & 14 & 3955 & 39,55 & 158,5 & 1500 & 15 \\
\hline corrida 4 & 14,1 & 3792 & 37,92 & 163 & 1501 & 15,01 \\
\hline corrida 5 & 14 & 4010 & 40,1 & 159,8 & 1810 & 18,1 \\
\hline corrida 6 & 14,2 & 4090 & 40,9 & 168,1 & 1500 & 15 \\
\hline Media & $\mathbf{1 4 . 1}$ & $\mathbf{3 8 8 3}$ & $\mathbf{3 8 , 8}$ & $\mathbf{1 6 1 , 5}$ & $\mathbf{1 6 9 9}$ & $1 \mathbf{1 7 , 0}$ \\
\hline
\end{tabular}

\begin{tabular}{|c|c|c|c|c|c|c|}
\hline \multicolumn{4}{|c|}{ Resultados con SAE J1349 prueba 2} & \multicolumn{3}{|c|}{ Gas } \\
\hline & $\begin{array}{l}\text { Potencia } \\
\text { maxima (hp) }\end{array}$ & $\begin{array}{c}\text { Régimen } \\
\text { máxima } \\
\text { potencia (rpm) }\end{array}$ & $\begin{array}{c}\text { Régimen } \\
\text { máxima } \\
\text { potencia (rpm } \\
\times 100)\end{array}$ & $\begin{array}{l}\text { Par Torsor } \\
\text { máximo }(\mathrm{Nm})\end{array}$ & $\begin{array}{l}\text { Régimen } \\
\text { máximo par } \\
(\text { (rpm) }\end{array}$ & $\begin{array}{l}\text { Régimen } \\
\text { máximo par } \\
(\mathrm{rpm} \times 100)\end{array}$ \\
\hline 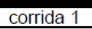 & 16,7 & 3613 & 36,13 & 185 & 2381 & 23,81 \\
\hline corrida 2 & 16,8 & 3840 & 38,4 & 194,8 & 1502 & 15,02 \\
\hline corrida 3 & 16.6 & 3955 & 39,55 & 188,6 & 1500 & 15 \\
\hline corrida 4 & 16,7 & 3792 & 37,92 & 194 & 1501 & 15,01 \\
\hline corrida 5 & 16.7 & 4010 & 40,1 & 190,2 & 1810 & 18.1 \\
\hline corrida 6 & 16.8 & 4090 & 40.9 & 199,1 & 1500 & 15 \\
\hline Mecdia & 16,7 & 3883 & 38,8 & 192,0 & 169 & 17. \\
\hline
\end{tabular}

Figura 6. Datos de potencia vs régimen de giro, sin inyección.

\section{d- Prueba con inyección de agua}

Similar a la prueba anterior, en las pruebas con inyección se hacen dos pruebas con seis repeticiones válidas, o sea que deben pasar por un control estadístico para que no se tengan altas dispersiones en la repetición.

\begin{tabular}{|c|c|c|c|c|c|c|}
\hline \multicolumn{4}{|c|}{ Resultados sin corrección prueba 3} & \multicolumn{3}{|c|}{ Gas Agua } \\
\hline & $\begin{array}{c}\text { Potencia } \\
\text { maxima (hp) }\end{array}$ & $\begin{array}{c}\text { Régimen } \\
\text { máxima } \\
\text { potencia }(\mathrm{rpm})\end{array}$ & \begin{tabular}{|c|} 
Régimen \\
máxima \\
potencia $(\mathrm{rpm}$ \\
$\times 100)$
\end{tabular} & $\begin{array}{c}\text { Par Torsor } \\
\text { máximo }(\mathrm{Nm})\end{array}$ & $\begin{array}{l}\text { Régimen } \\
\text { máximo par } \\
(\mathrm{rpm})\end{array}$ & $\begin{array}{l}\text { Régimen } \\
\text { máximo par } \\
(\mathrm{rpm} \times 100)\end{array}$ \\
\hline corrida 1 & 12,5 & 4746 & 47,46 & 137,7 & 1786 & 17,86 \\
\hline corrida 2 & 13 & 4331 & 43.31 & 138.1 & 1829 & 18.29 \\
\hline corrida 3 & 12,7 & 4637 & 46,37 & 141,9 & 1828 & 18,28 \\
\hline corrida 4 & 12,6 & 4666 & 46.66 & 145.8 & 1839 & 18.39 \\
\hline corrida 5 & $\frac{12,0}{12,9}$ & 4497 & 44,97 & 147.9 & 1800 & 18 \\
\hline corrida 6 & 12,8 & 4372 & 43,72 & 148,4 & 1813 & 18,13 \\
\hline Media & 12,8 & $\overline{4542}$ & 45,4 & 143,3 & 181 & 18,2 \\
\hline \multicolumn{4}{|c|}{ Resultados con SAE J1349 prueba 3} & \multicolumn{3}{|c|}{ Gas Agua } \\
\hline & $\begin{array}{c}\text { Potencia } \\
\text { maxima (hp) }\end{array}$ & $\begin{array}{c}\text { Régimen } \\
\text { máxima } \\
\text { potencia (rpm) }\end{array}$ & \begin{tabular}{|c|} 
Régimen \\
máxima \\
potencia (rpm \\
$\times 100)$
\end{tabular} & $\begin{array}{c}\text { Par Torsor } \\
\text { máximo }(\mathrm{Nm})\end{array}$ & $\begin{array}{l}\text { Régimen } \\
\text { máximo par } \\
\quad(\mathrm{rpm})\end{array}$ & $\begin{array}{l}\text { Régimen } \\
\text { máximo par } \\
(\mathrm{rpm} \times 100)\end{array}$ \\
\hline corrida 1 & 15 & 47 & 47.46 & 16 & 1786 & 17.86 \\
\hline corrida 2 & 15,5 & 4331 & 43,31 & 164,9 & 1829 & 18.29 \\
\hline corrida 3 & 15.2 & 4637 & 46.37 & 169 & 1828 & 18.28 \\
\hline corrida 4 & 15,1 & 4666 & 46,66 & 173,8 & 1839 & 18,39 \\
\hline corrida 5 & 15,4 & 4497 & 44,97 & 176 & 1800 & 18 \\
\hline corrida 6 & 15,2 & 4372 & 43,72 & 176.5 & 1813 & 18,13 \\
\hline Media & $\overline{15,2}$ & 4542 & $4 \overline{45,4}$ & 170,7 & 181 & $\overline{18,2}$ \\
\hline
\end{tabular}

Figura 7. Datos de potencia vs rpm, con inyección de agua.

\section{e- Prueba con inyección de agua - etanol.}

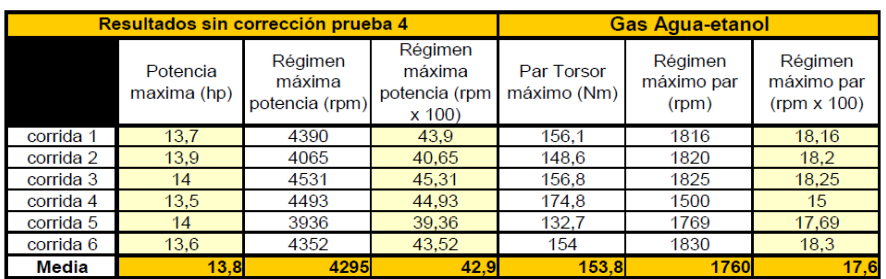

\begin{tabular}{|c|c|c|c|c|c|c|}
\hline \multicolumn{4}{|c|}{ Resultados con SAE J1349 prueba 4} & \multicolumn{3}{|c|}{ Gas Agua-etanol } \\
\hline & $\begin{array}{l}\text { Potencia } \\
\text { maxima (hp) }\end{array}$ & $\begin{array}{c}\text { Régimen } \\
\text { máxima } \\
\text { potencia }(\mathrm{rpm})\end{array}$ & $\begin{array}{c}\text { Régimen } \\
\text { máxima } \\
\text { potencia (rpm }\end{array}$ & $\begin{array}{c}\text { Par Torsor } \\
\text { máximo }(\mathrm{Nm})\end{array}$ & $\begin{array}{l}\text { Régimen } \\
\text { máximo par } \\
\text { (rpm) }\end{array}$ & $\begin{array}{l}\text { Régimen } \\
\text { máximo par } \\
\text { (rpm x 100) }\end{array}$ \\
\hline & & $\overline{43}$ & $\frac{40,9}{43,9}$ & & 1816 & $\overline{18,16}$ \\
\hline 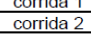 & 16,4 & & $\frac{43,9}{40,65}$ & $1 / 5,8$ & $\frac{1870}{1820}$ & $\frac{18,16}{18,2}$ \\
\hline corrida 3 & 16,6 & $\frac{45}{45}$ & & 4 & 1825 & 18.25 \\
\hline corrida 4 & & 4493 & & & 1500 & $\frac{15}{15}$ \\
\hline corrida 5 & 6,5 & 3936 & 39,36 & 156,6 & 1769 & 17,69 \\
\hline corrida 6 & $\frac{16}{16}$ & 4352 & 43.52 & $\frac{181.4}{1810}$ & 1830 & 18,3 \\
\hline
\end{tabular}

Figura 8. Datos de potencia vs rpm, con inyección de agua - etanol.

\section{f- Prueba con inyección agua - metanol}

\begin{tabular}{|c|c|c|c|c|c|c|}
\hline \multicolumn{4}{|c|}{ Resultados sin corrección prueba 5} & \multicolumn{3}{|c|}{ Gas Agua-metanol } \\
\hline & $\begin{array}{c}\text { Potencia } \\
\text { maxima (hp) }\end{array}$ & $\begin{array}{c}\text { Régimen } \\
\text { máxima } \\
\text { potencia }(\mathrm{rpm})\end{array}$ & $\begin{array}{c}\text { Régimen } \\
\text { máxima } \\
\text { potencia }(\mathrm{rpm} \\
\times 100)\end{array}$ & $\begin{array}{c}\text { Par Torsor } \\
\text { máximo (Nm) }\end{array}$ & $\begin{array}{l}\text { Régimen } \\
\text { máximo par } \\
(\mathrm{rpm})\end{array}$ & $\begin{array}{l}\text { Régimen } \\
\text { máximo par } \\
(\text { rpm } \times 100)\end{array}$ \\
\hline & 10,0 & 42 & 42,27 & & 1814 & 18,14 \\
\hline & 13,9 & 4294 & 42,94 & 172,2 & 1500 & 15 \\
\hline corrida 3 & 14 & 4330 & 43,3 & 160,1 & 1836 & 18,36 \\
\hline corrida 4 & $\overline{14,1}$ & 4522 & 45,22 & 164,7 & 1868 & 18,68 \\
\hline corrida 5 & 14 & 4090 & 40,9 & $\frac{171,9}{176.6}$ & 1500 & 15 \\
\hline corrida 6 & $14 \quad 14$ & $\frac{4056}{425}$ & 40,56 & $\frac{176,5}{1659}$ & 1500 & 15 \\
\hline
\end{tabular}

\begin{tabular}{|c|c|c|c|c|c|c|}
\hline \multicolumn{4}{|c|}{ Resultados con SAE J1349 prueba 5} & \multicolumn{3}{|c|}{ Gas Agua-metanol } \\
\hline & $\begin{array}{c}\text { Potencia } \\
\text { maxima (hp) }\end{array}$ & $\begin{array}{c}\text { Régimen } \\
\text { máxima } \\
\text { potencia (rpm) }\end{array}$ & $\begin{array}{c}\text { Régimen } \\
\text { máxima } \\
\text { potencia (rpm } \\
\times 100)\end{array}$ & $\begin{array}{c}\text { Par Torsor } \\
\text { máximo (Nm) }\end{array}$ & $\begin{array}{l}\text { Régimen } \\
\text { máximo par } \\
(\mathrm{rpm})\end{array}$ & $\begin{array}{l}\text { Régimen } \\
\text { máximo par } \\
(\mathrm{rpm} \times 100)\end{array}$ \\
\hline & & & 42.27 & 175.8 & 1814 & 18.14 \\
\hline orrida 2 & 16 & 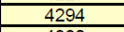 & 42,94 & 201,7 & 1500 & $\frac{15}{150}$ \\
\hline$\frac{\text { corrida } 3}{\text { corrida } 4}$ & $\frac{16,5}{16,5}$ & $\frac{4330}{4522}$ & $\frac{43,3}{45,22}$ & $\frac{187,6}{193.2}$ & $\frac{1836}{1868}$ & $\frac{18,36}{18,68}$ \\
\hline corrida 5 & $\frac{16,4}{16,4}$ & & 40,9 & 201,6 & 1500 & $\frac{15}{15}$ \\
\hline corrida 6 & 16.4 & 4056 & 40.56 & 207,1 & 1500 & 15 \\
\hline Media & 16, & 425 & & 194 & 1670 & \\
\hline
\end{tabular}

Figura 9. Datos de potencia vs rpm, con inyección de agua- metanol.

\section{g- Gráfico comparativo de potencia máxima}

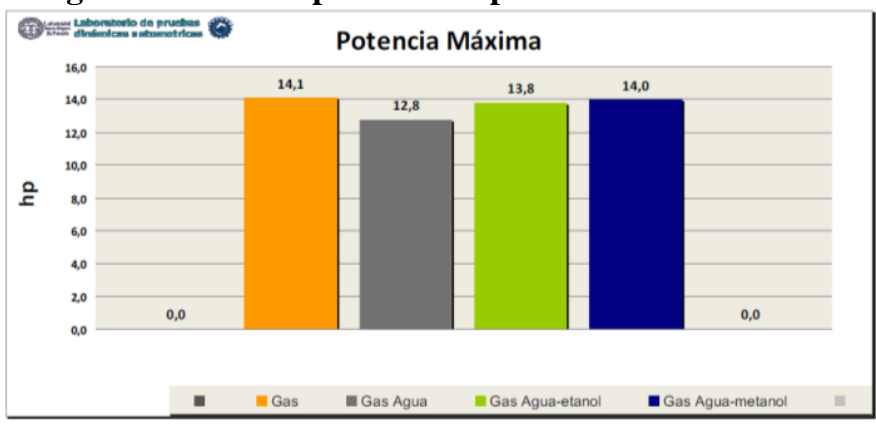

Figura 10. Potencia máxima comparativa. 


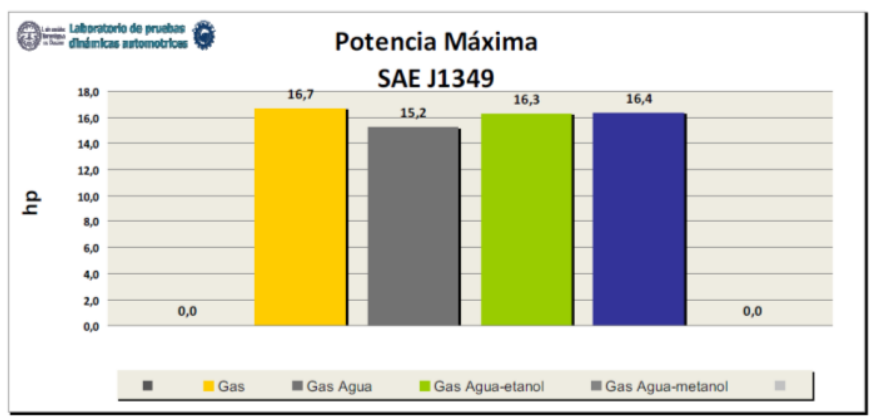

Figura 11. Potencia máxima comparativa, con corrección de condiciones ambientales Norma SAE J1349.

En el análisis de los datos obtenidos, se evidencia una disminución de la potencia del vehículo al utilizar el sistema de inyección de agua sin ningún tipo de mezcla (Es decir, únicamente agua como aditivo), comparado con los resultados obtenidos al funcionar el sistema sólo dedicado a gas natural sin el funcionamiento del dispositivo. Esta caída de potencia se encuentra alrededor de un $15 \%$, como consecuencia de las condiciones químicas y energéticas del agua, que es un fluido que actúa sólo como refrigerante pero en ningún momento como combustible que pudiera llegar a enriquecer la mezcla.

Es claro que la inyección de agua disminuye la eficiencia de quemado del gas.

\section{h- Gráficos comparativos de par torsor máximo}

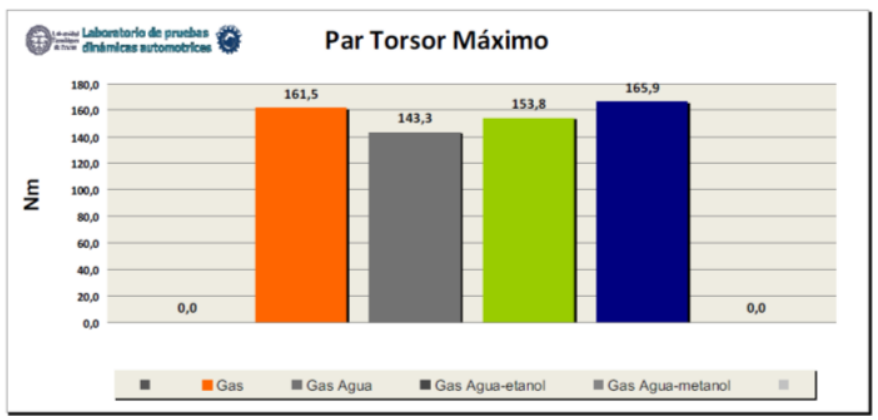

Figura 12. Par torsor máximo comparativo.

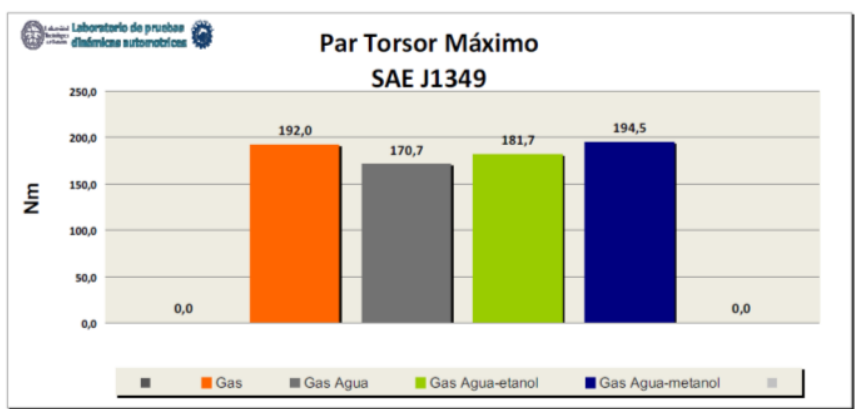

Figura 11. Par torsor máximo, con corrección de condiciones ambientales Norma SAE J1349.

El comportamiento del par torsor es similar al que presenta la potencia y los porcentajes mostrados son ligeramente menores. Este es un resultado esperado, ya que una variable depende de la otra.

Se presentan las gráficas de cambios de la potencia y par torsor con respecto al cambio del régimen de giro del motor.

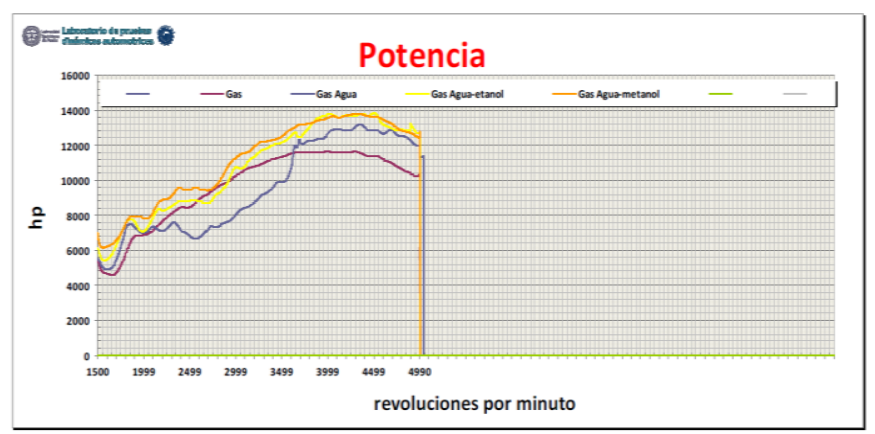

Figura 12. Potencia vs rpm

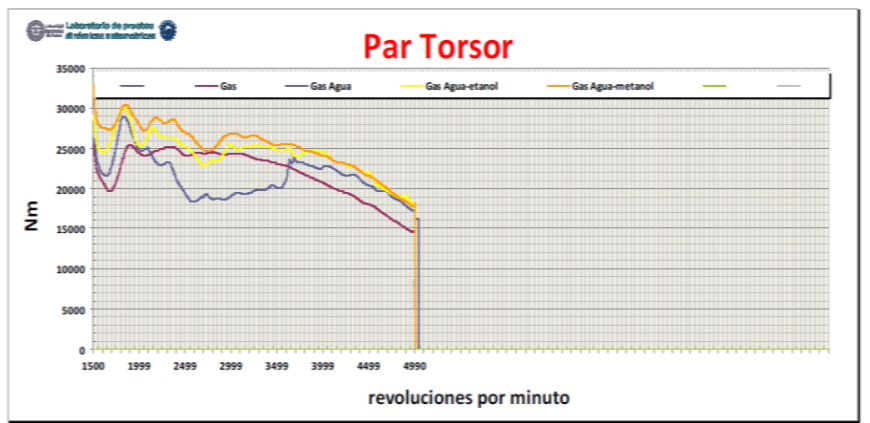

Figura 12. Par torsor vs rpm

Según los resultados obtenidos en esta parte usando mezclas agua etanol o agua metanol no se tiene una reducción significativa de la potencia para todo el régimen de revoluciones del motor, caso contrario a inyectar sólo agua; ya que se observa variación de la potencia en la prueba.

\section{i- Comparativo de temperaturas de gases de escape}

Para todas las pruebas realizadas se realizó un registro de la temperatura de salida de gases, muy cerca de la cámara de combustión.

En las siguientes gráficas se muestran el comportamiento de la temperatura en el tiempo de la prueba y los promedios comparativos obtenidos en las diferentes repeticiones de la prueba. 


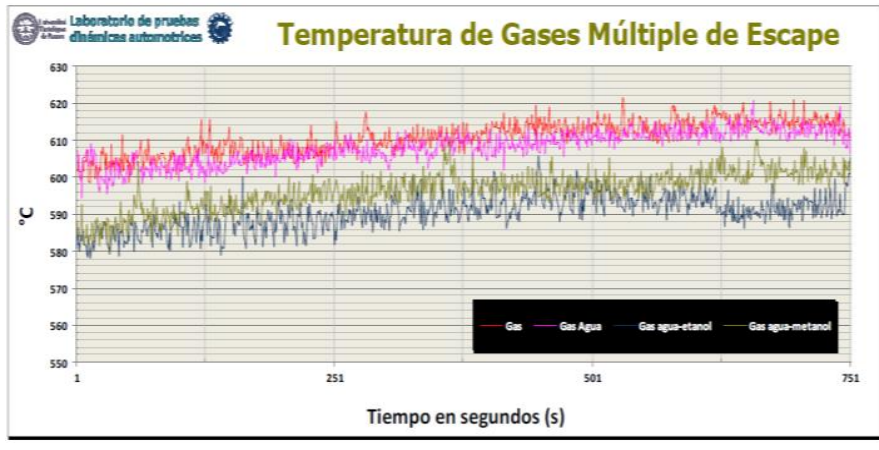

Figura 14. Resultados de temperatura

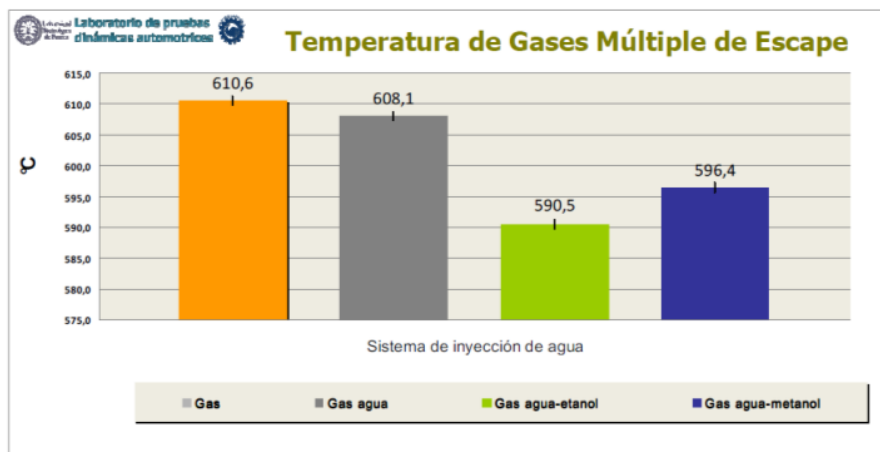

Figura 15. Promedios de temperatura

El análisis de los resultados de temperatura muestra un claro potencial de la mezcla agua - etanol para lograr disminuir la temperatura en la cámara de combustión del motor ensayado. Co este sistema de inyección y usando la mezcla agua - etanol, se logra mantener la potencia generada por el motor y se disminuye en $20^{\circ} \mathrm{C}$

La temperatura de los gases de combustión, lo cual representa una disminución del $3 \%$.

El dispositivo se mantendrá instalado en le vehículo en su trabajo normal de tal manera que se hará un seguimiento que permita concluir si es suficiente esta disminución en la temperatura.

\section{CONCLUSIONES}

- Haciendo uso del sistema de inyección de agua utilizando una mezcla de agua-etanol como aditivo, se garantiza una mejora importante en la temperatura de los gases de escape.

- La temperatura de los gases de escape medidos muy cerca de la cámara de combustión son confiables y concluyentes sobre la utilización del dispositivo de inyección para disminuir la temperatura del motor.

- La utilización del dispositivo de inyección de agua en el vehículo de prueba dedicado a gas natural como combustible, es viable: siempre y cuando se utilice la mezcla de agua-etanol y no recomendable para ser usado con sólo agua como aditivo, debido a que las pérdidas en potencia y par torsor son grandes.
- La permanencia en la potencia y el par tosor en las pruebas obtenidas en la utilización agua-etanol y agua-metanol se convierten en parte fundamental para los resultados y datos concluyentes respecto a la mezcla ideal que debe utilizar el dispositivo, a su vez de la viabilidad de su utilización en vehículos dedicados a gas natural.

- La metodología de pruebas usada demostró ser la adecuada para este tipo de investigación, quedando pendiente el análisis de los efectos de la inyección de refrigerante en los gases contaminantes y en la disminución de la eficiencia de la combustión.

\section{RECOMENDACIONES}

Realizar un análisis, desde la combustión, para explicar la pérdida de potencia observada cuando se usó agua en la inyección.

Hacer un análisis de los contaminantes en los gases de escape, que determinen el impacto en el medio ambiente al usar inyección de agua o agua - etanol en un motor de combustión interna.

Realizar este mismo estudio para un motor de mayor cilindraje que trabaje con gas natural, donde las altas temperaturas hacen que éstos tengan muchos problemas ligados al sobrecalentamiento de las partes internas del motor.

\section{$\underline{\text { Referencias de libros: }}$}

[1]. CASTRO, Miguel de; Trucaje De Motores De 4 Tiempos; SPAÑA: EDICIONES CEAC, 1963.

[2]. CHIGIER, N. Combustion measurements. Washington, Hemisphere Publishing Corp. 1991.

[3]. GILLIERI, Stefano, Preparación de Motores de Serie Para Competición; ESPAÑA: EDICIONES CEAC, 1994.

$\underline{\text { Referencias de publicaciones periódicas: }}$ NACIONAL

$\mathrm{DE}$ BIOCOMBUSTIBLES: http://www.fedebiocombustibles.com/

[5]. REVISTA ECOGAS, revista especializada del GNV $\mathrm{N}^{\mathrm{o}} 24$ abril del $20057 \mathrm{p}$.

Otras referencias:

[6]. MINISTERIO DE MINAS Y ENERGÍA / BIOCOMBUSTIBLES: http://www.minminas.gov.co/minminas/hidrocarburo 
s.jsp? cargaHome $=3 \&$ id categoria $=149 \&$ id subcateg oria $=228$

[7]. PELLEGRINI

RACING:

http://www.pellegriniracing.com.ar http://www.pellegriniracing.com.ar/tecnica.htm

[8]. PELLEGRINI, Humberto; Notas de clase: Preparación de motores atmosféricos y sobrealimentados; La plata, Buenos Aires Argentina; 2006.

[9]. STREET TUNNING COLOMBIA: http://www.stcolombia.com/forooficial/forumdisplay .php?fid=15

http://www.stcolombia.com/forooficial/viewthread.p $\underline{\text { hp?tid}=20144}$ 